\title{
Research on Health Assessment for Ecologic Restoration of Slope
}

\author{
Shu $\mathrm{Cao}^{1}$ and Feng Xiong, ${ }^{2, *}$ \\ ${ }^{1}$ NO. 710 R\&D Institute, CSIC, Yichang ,Hubei 443000,China \\ ${ }^{2}$ College of Civil Engineering \& Architecture, China Three Gorges University, Yichang, Hubei 443000, China \\ *Corresponding author
}

\begin{abstract}
Environment problems have drawing more and more attention in recent years,the ecologic restoration engineering of slope have progressively increased too.As a result, the engineering is needed to be assessed. Now, a lot of methods which macroscopicallyassesse that in all respects are used, but this paper only researches the assessment method that is aimed at ecological health of the engineering. Firstly, the ecological health of vegetation restoration is defined, then specific indexes are selected from ecosystem stability, resistance and community successionaccording toabovedefinition. At last, the weight of each index is determined,and the assessment resultof each index and the final assessment result is figured out. This assessment method is more targeted and objective for artificialecological remediation of slope.
\end{abstract}

Keywords-slope;ecological restoration; ecological health;analytic hierarchy process

\section{INTRODUCTION}

In recent years, Chinese infrastructure has developed quickly, the ecological environment of the area where the engineering locals in has been destroyed inevitably. Artificial slope is one of the common destruction of water conservancy construction and road construction. Bare slopes lost the soil matrix for plant growth and increased probability of slope instability, which will easily cause soil erosion or even landslide, debris flow and other disasters [1]. It must be controlled, and side slope ecology repair technical which utilize the principle that vegetation retains water and soil for slope stability and beautifying surroundings is a multidisciplinary and comprehensive engineering technology. Its advantage lies in combining slope protection and ecological restoration and landscape constructiontogether[2]. Now there are a lot of engineering of slope ecologic restoration, and with the implementation of "thirteen five" economic plan, there will be an increasing number of the engineering of slope ecologic restoration. So the assessment of these engineering's result will attract more and more attention.

Many scholars have researched and proposed corresponding assessment system about how to assess the effect of completed slope ecological restoration. For example, based on Analytic Hierarchy Process (AHP) , Sheng Fang(2008) established the assessment system about quality of vegetation slope protection through analyzing the relevant theoretical which include vegetation slope protection, ecology, aesthetics theory of landscape and so on[3]. According to ecology, presentation quality and mechanical index,
HaohaoJiang(2009) assessed the effect of slope vegetation protection by using grey correlation degree method and principal component analysis respectively, and the conclusion of this two method was similar[4]. Many of the researches are based on AHP, and get the index weights through expert consulting so as to assessing the effect of slope ecologic restoration quantitatively and qualitatively with certain rationality[5]. But many of these assessment systems aim at the whole effect and lack of thorough research for a class of these indicators especially the health of ecosystem. So this paper will set up assessment system for slope ecologic restoration.

\section{The Definition of Health Assessment}

Ecosystem health is a systematic diagnostic mode for ecosystem state characteristics based on the framework of ecology combining with human health. But domestic and foreign scholars have not reached a consensus about the exact definition[6]. Health is a relative concept, there is no absolutely healthy ecosystem as well as health standard[7]. This paper give the definition of health assessment for artificial slope as follow: after the ending of artificial maintenance, the ecosystem of slope is stable and resistant, besides showing the trend of positive community succession. Health assessment is a way to quantify health status of ecosystem based on related indicators and related data combined with definition of health assessment.

\section{EVALUATION INDEXES SELECTION}

This paper selects the 2-level indicators from ecosystem stability, resistance, and trend of community succession combining with definition of health assessment.

\section{A. Ecosystem Stability}

Ecosystem is a general designation which includes the biology and environment in a specific area. The part of life includes producers, consumers and decomposers. The three makes exchange with environment about material and energy, life and environment form a whole by flow of material and energy, in that case the ecosystem can be stable. The artificial slope's ecosystem is almost complete, so this paper selects soil fertility, soil microbial content, vegetation coverage and soil animal as the indexes from these aspects.

- soil fertility is the guarantee for vegetation to grow well and shows the integrated features of soil. It includes soil's physical properties such as texture and chemical properties such as acidity, mineral element, 
and visible features such as biological activity, erosion. Those are important factors for maintaining the sustainable development of ecosystem[8].

- Most of microorganism exist as decomposers which is the indispensable part of ecosystem. most of them can be found in soil for the ecosystem of artificial slope. Jing Li (2012) and many scholars' research shows that the higher the diversity of soil microbial community is, the more stable of soil ecosystem will be[9]. So soil microorganism is the necessary condition of stability and health of slope ecosystem.

- Vegetation coverage reflects the effect of ecological restoration directly and the growth of vegetation, which is the most important in the ecosystem as producer.

- Soil animal is an indispensable part in the ecosystem as consumer. The ecosystem of artificial slope is very open, many animals have greater mobility especially for the flying animal. Soil animal is the fauna which has an effect on soil and lives in soil environment including large plant residues regularly or temporarily[10]. It has less mobility, so this paper selects it as index in consideration of the feasibility of statistics.

\section{B. Resistance}

Being at the initial stages of ecological restoration, artificial plants would suffer drought, saline-alkali, high or low temperature and many adverse conditions during the growth. On one hand, it would influence plants' growth and development and lead to death of plants in serious case. On the other hand, plants have evolved a series of mechanism to resist the adverse conditions during the adversity, that is the resistance[11]. This paper selects three representative stresses which are drought, salt and high or low temperature to assess.

- Drought stress will arise when soil moisture is below what the plants need for a long time, plants' morphology, osmotic adjustment and so on will change accordingly. The number of leaves of plants and leaf area will reduce, and plants will dwarf under drought stress. The drought stress is also the most common stress, so the plants that are used for ecological restoration must have drought resistance.

- $\quad$ Salt stress will arise when the soil salt content ranges from $0.20 \%$ to $0.25 \%[12]$. Plants' leaf area and tiller number will reduce under salt stress. Salt stress is difficult to solve artificially. So the plants that are used for ecological restoration must have salt resistance.

- Temperature stress will arise when the temperature is too lower or higher than normal temperature that plants grow in for a long time. Plants will appear wilt, drying out, crisping, leaf abscission under temperature stress. So the plants used for ecological restoration must have heat and cold resistance considering the Chinese climate characteristic.

\section{Community Succession Tendency}

Most of the plants used for ecological restoration are herbs, they are growing vigorously in the early stages of restoration. But the best result of restoration is that plants community succession occurred and returned to its natural state. This paper selects woody plants and species diversity as the indices from community succession tendency.

- The order of healthy slope ecological succession trend is that evolving from herbs to shrubs and last trees, succeeding into stable climax. But the stage researched in this paper is the beginning of succession, it is crucial. The shrubs have formed scale and the trees should began to grow in this stage. So select the number and species of woody plants as the index.

- Complex ecosystem is the most stable and it has great self-organization. But the species of ecosystem during early successional stages is simple. So species diversity can reflect the community succession tendency for the early artificial slope's ecosystem and select it as the index.

\section{Assessment MethoD}

\section{A. Determine the Weights}

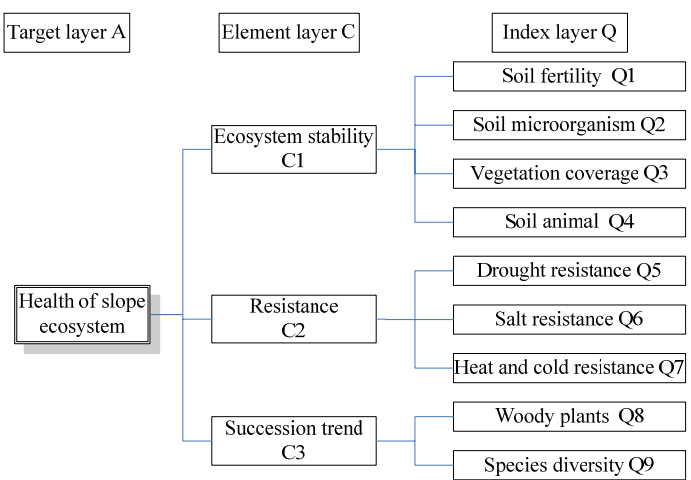

FIGURE I. THE HIERARCHICAL CHART OF ASSESSMENT SYSTEM

All the 2-level indexes' weight are determined by the analytic hierarchy process (AHP), the hierarchical chart is shown in figure 1.

First, pundits are invited by researcher, one of this field's pundit compares the importance of elements C1, C2, C3 with each other for target layer using 1-9 scale method, structures the judgment matrix A. Pundit compares the importance of indexes Q1, Q2, Q3,Q4 with each other for element C1, structures the judgment matrix $\mathrm{C} 1$, structures judgment matrix C2, C3 by the same method. Then researcher calculates every judgment matrix's eigenvector $\mathrm{W}$ and first eigenvalue $\lambda_{\text {max }}$ by sum method of fuzzy mathematics, and makes consistency examination for every judgment matrix. If there is judgment matrix that couldn't pass the consistency examination, it should be structured again by the above method. Last, researcher makes hierarchial population ordering and combined consistency examination by the method of fuzzy mathematics, if it can't pass the examination, the above steps would be repeated until it could pass. All indexes' weights will have been got. 
There are number of pundits, and the indexes' weights are got from every pundit by above method. Researcher calculates average of the indexes' weights from every pundit. The indexes' final weights $a_{1}, a_{2}, \cdots, a_{9}$ are got.

\section{B. Determine Indexes' Score}

1)Sample plots:Artificial slope is divided into $1 \mathrm{~m} \times 1 \mathrm{~m}$ grid, the investigator selects $\mathrm{n}$ grids randomly as sample plots, total area of the $n$ grids occupies $1 \%$ of total artificial slope. Investigator selects 5 soil samples randomly from every sample plots.

2)Control samples:Some of the indexes are assessed by comparing. A healthy local natural slope is similar to the artificial slope which is needed. Investigator selects 5 areas of $1 \mathrm{~m} \times 1 \mathrm{~m}$ size randomly as the control samples, and selects 5 soil samples randomly from every control samples.

3) Calculate the score of indexes:

a) Investigator analyzes the soil of all sample plots to get the soil fertility index (SFI) that is the soil fertility's score $q_{1}$.

b) Investigator determines the microorganism populations of all soil samples denoted by $b_{1}$ and determines the microorganism populations of all control soil samples denoted by $b_{2}$, the score of soil microbial content is denoted by $q_{2}=\frac{b_{1}}{b_{2}}$.

c) Investigator takes HD photography for all areas of artificial slope and analyzes the vegetation coverage by image analysis, the coverage is the score of vegetation coverage $q_{3}$.

d) Investigator determines the animal number in soil of all soil samples denoted by $c_{1}$, then determines the animal number in soil of all control soil samples denoted byc $c_{2}$, the score of soil animal is $q_{4}=\frac{c_{1}}{c_{2}}$.

e) Investigator counts the number of plants which have dwarfed and its number of leaves, leaf area have reduced at all sample plots denoted by $d_{1}$ and counts the sum of plants at all sample plots denoted byd.The score of drought resistance is $q_{5}=1-\frac{d_{1}}{d}$. The scores of salt resistance and heat and cold resistance are got by the same method successively denoted by $q_{6}$ and $q_{7}$.

f) Investigator counts the number of woody plants at all sample plots denoted by $e_{1}$, the score of woody plants is $q_{8}=\frac{e_{1}}{d}$.

g) Investigator counts the species and quantity, and measures the species diversity by Shannon-Wiener index, the score is $q_{9}=-\sum_{i=1}^{S} p_{i} \log _{2} p_{i}$.

\section{Assessment Result}

The final score of assessment equals to the sum of every index's score multiply its weight.

$$
\mathrm{A}=q_{1} \cdot a_{1}+q_{2} \cdot a_{2}+\cdots+q_{9} \cdot a_{9}(1)
$$

A is the final score, but health is a relative concept. A lot of practice of assessment is to be needed to get one standard what is healthy ecosystem of artificial slope.

\section{SUMMARY AND Prospect}

This paper researches the assessment for ecological health of artificial slope meticulously based on previous research. This method assesses the ecological health through ecosystem stability, resistance and trend of community succession. Three elements including nine 2-level indexes based on analytic hierarchy process (AHP), embodies the characteristic of artificial slope. The advantage of this method is that all data is objective except indexes' weight and the subjectivity of indexes' weight is reduced by AHP. The shortcoming of this method is the assessment of resistance that lacks the support of scientific theory and this method can't determine the assessment grade by scientific analysis. These are needed to be researched and improved further.

\section{REFERENCES}

[1] Fangshuai Dong,Ligen Xu.The Establishment of Ecological Assessment Indexes System for Petrous Side Slope Revegetation Engineering[J].Bulletin of Science and Technology.2009, 25(4):503-514.

[2] Shiqi Liu.Study on Bionegineering Tecniques for Slop Protection and Comprehensive Protection System[D].Wuhan:Institute of Rock and Soil Mechanics the Chinese Academy of Sciences,2004:1-5.

[3] Sheng Fang.Research on Quality Evaluation Method of Slope Vegetation[D].Xi'an:South West Jiaotong University,2011.

[4] Haohao Jiang.Effect evaluation of slope protection for herbs based on the grey connection and principal component analysis[D].Harbin:Northeast Forestry University,2009.

[5] Bailei Zhao.Research on quality control and effect evaluation Method of ecological slop protection in mountain area expressway[D]. Chongqing:Chongqing Jiaotong University,2014.

[6] Jian Peng,Yanglin Wang,Jiansheng Wu,Yuqing Zhang.Evaluation for regional ecosystem health: methodology and research progress[J].ActaEcologica Sinica,2007,27(11):4877-4885.

[7] Yihan Wang,Demin Zhou,Yonghua Sun.Assessment of the ecological health of wetlands in Honghe supported by RS and GIS techniques.Acta Ecologica Sinica,2011,31(13):3590-3600

[8] Pathak P;Sahrawat K.L;Rego T.J.etal.Measurablebiophysical indicator for impact assessment:changesin soilquality[M].ShiferawB.,FreemanH.A.SwintonS.M.Natural Resource Management in Agriculture, Methods for Assessing Economicand Environmental Impacts.UK:Crom-wellPress,2004:53- 74.

[9] Jing Li,Yurong Liu,Jizheng He,Yuanming Zheng.Insights into the responses of soil microbial community to the environmental disturbances[J].Acta Scientiae Circumstantiae, 2013,033(4):959-967.

[10] Haitao Wu,Xianguo Lu,Qing Yang,Ming Jiang.Ecolcogical characteristics and functionns of soil fauna community[J].Acta Pedologica Sinica,2006,42(2):314-323.

[11] Xiuchen Chen,Dongjin Xiong.Research Progress in Plant Stress Resistance[J].Hubei Agricultural Sciences,2010,49(9):2253-2256.

[12] Hongfei Qi,Xiaocheng Yang. Review of the Research on Plant Stress Resistance[J].Journal of Anhui Agricultural Sciences,2008,36(32):13943-13946. 